Morales-Ortiz, E.; Burgueño, R.; Cueto-Martín, B.; Macarro-Moreno, J.; Medina-Casaubón, J. (2021) Can Sport Education Improve Attitudes towards Secondary Physical Education? Revista Internacional de Medicina y Ciencias de la Actividad Física y el Deporte vol. 21 (84) pp. 435-450. Http://cdeporte.rediris.es/revista/revista83/artpueden1267.htm

DOI: https://doi.org/10.15366/rimcafd2021.83.002

\title{
ORIGINAL
}

\section{¿PUEDE LA EDUCACIÓN DEPORTIVA MEJORAR LAS ACTITUDES HACIA LA EDUCACIÓN FÍSICA EN SECUNDARIA?}

\section{CAN SPORT EDUCATION IMPROVE ATTITUDES TOWARDS SECONDARY PHYSICAL EDUCATION?}

\author{
Morales-Ortiz, E. ${ }^{1}$; Burgueño, R. ${ }^{2}$; Cueto-Martín, B. ${ }^{\text {; }}$ Macarro-Moreno, J. ${ }^{1}$ \\ y Medina-Casaubón, J. ${ }^{1}$ \\ ${ }^{1}$ Doctores en Educación Física. Departamento de Educación Física y Deportiva, Universidad de \\ Granada (España) esthermo@ugr.es, belencueto@ugr.es, jmacarro@ugr.es, jmedinac@ugr.es \\ 2 Doctor en Educación. Departamento de Educación Física, Facultad de Ciencias de la Salud, \\ Universidad Isabel I (España) rafael.burgueno@ui1.es
}

Código UNESCO I UNESCO code: 5899 Educación Física y Deporte /

Physical Education and Sport.

Clasificación Consejo de Europa / Council of Europe classification: 4. Educación Física y Deporte comparado / Compared Sport and Physical Education; 5. Didáctica y metodología / Didactics and methodology.

Recibido 7 de julio de 2019 Received July 7, 2019

Aceptado 4 de noviembre de 2019 Accepted November 4, 2019

\section{RESUMEN}

La presente investigación tuvo como objetivo analizar la influencia de una temporada de Educación Deportiva sobre las actitudes hacia la Educación Física (EF) en estudiantes. Los participantes fueron 53 ( 31 hombres y 22 mujeres; $M_{\text {edad }}$ $=15.13, D T_{\text {edad }}=1.40$ ) estudiantes de secundaria quienes tomaron parte en una intervención de Educación Deportiva de 14 sesiones de 55 minutos. Se adoptó un diseño cuasi-experimental sin grupo control y medidas pre-test y post-test. Los resultados del MANOVA mostraron un aumento significativo en el nivel de valoración de la EF y su profesorado, dificultad de la EF, utilidad de la EF y preferencia por la EF en el alumnado. No hubo efectos multivariantes significativos respecto a la edad, género y deporte extraescolar. Los hallazgos son debatidos subrayando la importancia de la Educación Deportiva en el desarrollo de las actitudes del alumnado hacia la EF como un medio para favorecer su formación integral. 
PALABRAS CLAVE: práctica basada en modelos, modelos pedagógicos, enseñanza de la Educación Física, dominio actitudinal, currículum y enseñanza.

\section{ABSTRACT}

The objective of this research was to examine the influence of a Sport Education season on students' attitudes towards Physical Education (PE). The participants were 53 (31 male and 22 female; $\left.M_{\text {age }}=15.13, S D_{\text {age }}=1.40\right)$ secondary school students who took part in a Sport Education intervention consisting of fourteen 55-minute sessions. A quasi-experimental design with no control group and with pre-test and post-test measures was adopted. The results derived from MANOVA test showed a significant increase in the levels of perception of PE and the teacher, difficulty of PE, usefulness of PE and preference for PE in students after a Sport Education season. No significant multivariate effects were found for age, gender or after-school sport. These findings are discussed highlighting the importance of Sport Education in the development of the students' attitudes towards PE as a means to promote their comprehensive education.

KEYWORDS: model-based practice, pedagogical models, Physical Education teaching, attitudinal domain, curriculum and teaching.

\section{INTRODUCCIÓN}

Una meta frecuentemente aclamada para la Educación Física (EF) es el desarrollo actitudinal en el alumnado durante su proceso de enseñanzaaprendizaje (SHAPE America- Society of Health and Physical Educators, 2014). Las actitudes se conceptualizan, desde el área de la psicología social, como un conjunto de creencias, sentimientos y tendencias que predisponen a un individuo a actuar de un modo determinado. De esta manera, el individuo (e.g. estudiante) realiza una evaluación o juicio global de naturaleza positiva, neutra o negativa y, relativamente estable sobre cualquier aspecto de la realidad (e.g. iguales, ideas, cosas o la EF), el cual es entendido como un objeto de actitud (Briñol et al., 2002).

Los pedagogos de la EF han concebido a las actitudes como predisposiciones, ideas, miedos y convicciones personales hacia la asignatura de EF (Aicinena, 1991; Campbell, 1969). En concreto, Moreno-Murcia et al. (2003) operativizaron las actitudes hacia la EF en siete dimensiones diferentes: valoración de la EF y su profesorado (i.e. importancia atribuida por el alumnado a la asignatura y personal docente), dificultad de la EF (i.e. grado de complejidad de la EF en comparación con las otras asignaturas curriculares), utilidad de la EF (i.e. validez de la asignatura y sus contenidos en la formación integral del estudiante), empatía con la EF y con su profesor (i.e. compresión por parte del alumnado de la situación del docente y de la asignatura), concordancia con la organización de la EF (i.e. interpretación del alumnado sobre la estructura interna de la asignatura), preferencia por la EF (i.e. interés del alumnado sobre la actividad física) y EF como deporte (i.e. relación entre el deporte y la EF escolar). 
Varias investigaciones desarrolladas con el propósito de mejorar las actitudes hacia la EF, mediante intervenciones basadas en multi-actividades, han sido alentadoras dado que indicaron que era posible mejorar el nivel de las actitudes mostrado por el alumnado. En concreto, los trabajos informaron que se produjo un aumento en las actitudes hacia la EF cuando el alumnado participó en actividades personalizadas de condición física y manejo e interpretación de pulsómetros (Marttinen et al., 2018), en circuitos de habilidad por grupos (Digelidis et al., 2003), así como en actividades de resolución de problemas (GilMadrona et al., 2016). De igual manera, intervenciones que proporcionaron elección en las actividades, utilizando estilos de enseñanza reproductivos, y basados en tareas cooperativas tuvieron éxito a la hora de incrementar el nivel de actitudes del alumnado hacia la EF (Gülay et al., 2010).

Recientemente, los investigadores han hipotetizado que la promoción del dominio actitudinal del alumnado podría ser abordado por medio de la Educación Deportiva (ED) (Hastie \& Wallhead, 2016). En este aspecto, la ED es un modelo pedagógico centrado en el estudiante diseñado en torno a la educación del juego (Siedentop, 2002), dirigido primariamente a la enseñanza del contenido curricular del deporte en clase de EF (Siedentop et al., 2020). El objetivo primordial de la ED es crear una experiencia deportiva auténtica para el alumnado dentro de la asignatura de EF. Las principales metas de este modelo pedagógico son mejorar de manera efectiva el nivel de competencia, alfabetización y entusiasmo del estudiante en relación con la actividad deportiva. Para lograr tales metas, los estudiantes que participan en ED no sólo deben ser jugadores competentes, sino que deben ser capaces de demostrar alfabetización deportiva mostrando que valoran, entienden y aprecian el deporte, además de ser capaces de mostrar su entusiasmo hacia el deporte promocionando una cultura deportiva positiva (Siedentop et al., 2020).

Los objetivos y metas de la ED son logrados en el marco de experiencias deportivas significativas con estudiantes participando en temporadas deportivas modificadas, las cuales tienen una mayor duración que las unidades didácticas de EF tradicionales (Siedentop, 2002; Siedentop et al., 2020). La competición regular, que generalmente requiere que los equipos compitan en juegos o situaciones modificadas, sirve como base de estas temporadas. Para asegurar un fuerte sentido de afiliación al equipo, los estudiantes permanecen en equipos establecidos durante toda la temporada. Con la finalidad de hacer cada temporada auténtica y festiva, el alumnado registra y publica regularmente estadísticas individuales y de equipo, participa en un evento culminante y, realiza otros roles específicos además del rol de jugador (e.g. entrenador, árbitro o preparador físico) (Siedentop, 2002; Siedentop et al., 2020).

La ED parece ser un modelo pedagógico ampliamente vinculado con la mejora del dominio cognitivo, físico-motor y actitudinal del alumnado en el proceso de enseñanza-aprendizaje deportivo que tiene lugar en clase de EF (Araujo et al., 2014; Evangelio et al., 2018; Hastie et al., 2011). En lo referente al dominio cognitivo, la investigación previa ha apuntado que mientras que una temporada de ED facilitó la internalización por parte del alumnado de las características estructurales que definen a este modelo basado en la práctica (Burgueño et al., 2020), Wahl-Alexander et al. (2017) informaron de la necesidad de llevar a cabo 
varias temporadas de ED consecutivas para consolidar la internalización de los roles y desarrollar un conocimiento más profundo de este modelo pedagógico junto con un mayor sentido del juego limpio por parte del alumnado. De igual manera, la participación en una temporada de ED ha promovido la adquisición tanto de conocimientos relacionados con la condición física (Ward et al., 2017) como conocimientos vinculados con el reglamento deportivo (Ginciene \& Matthiesen, 2017; Mahedero et al., 2015), independientemente del género y el nivel de habilidad del estudiante (Pereira et al., 2016). En concreto, Farias et al. (2017) destacaron la existencia de un intercambio del conocimiento entre los estudiantes más habilidosos y aquellos menos habilidosos a la hora de perseguir objetivos de equipo de naturaleza más inclusiva. Asimismo, una temporada de ED favoreció el proceso de toma de decisiones del alumnado tanto en las situaciones reducidas de juego como en la competición formal (Mahedero et al., 2015).

Respecto al dominio físico-motor vinculado con el proceso de enseñanzaaprendizaje en clase de EF, una temporada basada en ED ha mejorado el nivel de condición física en el alumnado de educación secundaria (Ward et al., 2017), así como el nivel de resistencia aeróbica en el alumnado universitario (WahlAlexander \& Chomentowski, 2018). Por otra parte, los trabajos han mostrado la mejora del nivel de competencia percibida del alumnado después de una temporada de ED (Burgueño et al., 2018; Cuevas et al., 2015; Mesquita et al., 2016). En esta misma línea, Mahedero et al. (2015) descubrieron un aumento del nivel de rendimiento en el juego en el alumnado de educación secundaria después de una temporada de ED; mientras que Pereira et al. (2015) apuntaron un incremento en el rendimiento técnico en lanzamiento de peso, carrera de vallas y salto de longitud. No obstante, Hastie et al. (2017) informaron, en términos de eficacia de juego, que el alumnado menos habilidoso estuvo en desventaja cuando participaba junto con los estudiantes más habilidosos, lo cual fue más evidente en el caso de las alumnas. En cambio, los trabajos previos han señalado la mejora tanto del rendimiento técnico y táctico como de la implicación en el juego tras tres temporadas consecutivas basadas en ED (Farias et al., 2019).

En relación con el dominio actitudinal en clase de EF, los estudios previos han señalado consistentemente el incremento en el nivel tanto de compromiso como de implicación en el juego por parte del alumnado tras la implementación de la ED (Calderón et al., 2013; Farias et al., 2018). En concreto, Méndez-Giménez et al. (2016) descubrieron que, independientemente del nivel de habilidad del estudiante, el uso de material auto-construido promovió la implicación y el compromiso del alumnado en comparación con el empleo de material convencional en situaciones basadas en la ED. En esta misma línea, Layne y Hastie (2016) informaron de un aumento en el nivel de diversión en el alumnado después de una temporada de ED; mientras que Chu y Zhang (2018) junto con Martínez de Ojeda et al. (2021) encontraron un aumento de la motivación intrínseca en estudiantes. Es más, una temporada de ED favoreció el desarrollo de un comportamiento más ético en el alumnado medido por medio de la responsabilidad, la inclusión y el juego limpio (Harvey et al., 2014; WahlAlexander et al., 2017), además del fomento del respeto por los oponentes, reglas y árbitros (Méndez-Giménez et al., 2015). 
No obstante, hasta el momento, no hay evidencias de trabajos que hayan abordado la influencia de la ED sobre las actitudes del alumnado hacia la EF; a pesar de su importancia atribuida a la hora de favorecer el compromiso del estudiante con la clase de EF, de promocionar el nivel de actividad deportiva extraescolar y de consolidar un estilo de vida activo en edades posteriores (Gonzalez-Cutre et al., 2014; Sicilia et al., 2015). En esta misma línea, los pedagogos de EF consideran las actitudes hacia la EF como un adecuado medio para mejorar y pulir el andamiaje de los modelos pedagógicos con la finalidad de maximizar el aprendizaje cognitivo, físico-motor, socio-emocional y actitudinal del alumnado en clase de EF y, de este modo, fomentar su formación integral (Siedentop, 2002).

Por tanto, el objetivo del estudio fue analizar la influencia de la ED sobre las actitudes hacia la EF en alumnado de educación secundaria obligatoria durante el proceso de enseñanza-aprendizaje deportivo que tiene lugar en EF. Este estudio hipotetizó que una intervención basada en la ED aumentaría significativamente el nivel de la actitud de valoración de la EF, dificultad de la EF, utilidad de la EF, empatía con el profesor, concordancia, preferencia por la EF y la EF como deporte, respectivamente entre el inicio y el final del programa de intervención.

\section{MÉTODO}

\subsection{Participantes}

Los participantes fueron 53 estudiantes, (31 hombres y 22 mujeres) con edades entre 13 y 18 años $\left(M_{\text {edad }}=15.13, D T_{\text {edad }}=1.40\right)$ de cuarto curso educación secundaria obligatoria de un centro educativo público de una ciudad en el sureste español. De entre todos los participantes, 37 (69.81\%) estudiantes afirmaron practicar actividad deportiva extraescolar con una frecuencia semanal entre 1.50 horas y 22.00 horas $\left(M_{\text {frecuencia }}=4.77\right.$ horas, $D T_{\text {frecuencia }}=3.76$ horas). Ninguno de los participantes declaró tener experiencia previa con la ED. La técnica de muestro intencional fue utilizada a la hora de seleccionar los participantes del presente trabajo dada la facilidad de acceso a los centros educativos que tomaron parte.

\subsection{Instrumentos}

2.2.1. Actitudes hacia la Educación Física. Se utilizó el Cuestionario de Actitudes hacia la Educación Física desarrollado por Moreno-Murcia et al. (2003). Consiste en 56 ítems para medir la actitud de valoración de la EF y su profesor mediante 11 ítems (e.g. "Considero que el profesor se esfuerza en conseguir que mejoremos"), dificultad de la EF mediante 6 ítems (e.g. "Las actividades de EF son fáciles"), utilidad de la EF mediante 10 ítems (e.g. "Para mí es muy importante la EF"), empatía con el profesor y con la asignatura mediante 6 ítems (e.g. "El profesor de EF se preocupa más por nosotros que los demás profesores"), concordancia con la organización de la asignatura mediante 5 ítems (e.g. "Prefiero las clases prácticas a las clases teóricas"), preferencia por 
la EF y el deporte mediante 4 ítems (e.g. "Prefiero hacer algún deporte antes que ver la televisión") y la EF como deporte mediante 4 ítems (e.g. "En clases de EF sólo practicamos deporte"). De igual manera, hay una serie de ítems neutros que no se alinean dentro de ninguna dimensión de actitudes. Las respuestas a cada ítem se recogen en una escala Likert de 5 puntos, desde 1 (en desacuerdo) a 5 (totalmente de acuerdo).

\subsection{Diseño y Procedimiento}

De acuerdo con la investigación previa especializada en ED (Ginciene \& Matthiesen, 2017; Layne \& Hastie, 2016; Mahedero et al., 2015), el diseño fue un estudio cuasi-experimental sin grupo control y medidas pre-test y post-test (Ato et al., 2013). La presente investigación fue aprobada tanto por el Comité de Ética de la Universidad de Granada como por el centro educativo participante. Además, se dispone del consentimiento informado de los progenitores/tutores legales de cada estudiante participante. Tras plantear el programa de intervención, se llevó a cabo el pre-test, mientras que el post-test tuvo lugar una vez concluido dicho programa. Para ambas recogidas de datos, se utilizó un cuestionario que respetaba el anonimato y la voluntariedad de los sujetos en su compleción. El personal investigador estuvo a disposición del alumnado para resolver las dudas surgidas durante este proceso. El tiempo aproximado fue de 20 minutos.

\subsection{Programa de intervención}

2.4.1. Intervención de Educación Deportiva. La duración de esta intervención fue de 14 sesiones de 55 minutos, dos sesiones por semana durante siete semanas en horario habitual de EF. La duración de esta intervención estuvo en consonancia con la investigación previa (Calderón et al., 2013).

La intervención se dividió en cuatro fases. La primera de ellas fue la fase inicial, que consistió en una sesión introductoria donde cada profesor presentó las características de este modelo pedagógico, mientras que el alumnado se organizó en cuatro equipos de cinco o seis jugadores, se asignaron un rol específico a cada integrante (i.e. entrenador, preparador físico, árbitro, encargado del material, reportero), además de rellenar su ficha de equipo, elegir su color de vestimenta, escudo y lema. La segunda fase constó de tres sesiones de práctica dirigida por el profesor, donde el alumnado se familiarizó con la metodología de trabajo de este modelo pedagógico y se abordaron los aspectos técnico-tácticos básicos de los deportes alternativos a trabajar. La tercera etapa de siete sesiones fue la fase de práctica dirigida por el alumnado. En esta etapa se realizaron dos sesiones en las que el profesor marcaba unos objetivos de trabajo y los entrenadores eran los encargados de llevarlos a cabo y otras dos sesiones en las que además de llevar a cabo los objetivos marcados en la sesión había un tiempo de trabajo denominado Duty team. En esta etapa el docente se encargaba de dar feedback y animar al alumnado. Tanto en la fase de práctica dirigida por el profesor como en la dirigida por el alumnado, la sesión se organizó en: a) 10 minutos de calentamiento; b) 40 minutos de parte principal, donde se desarrollaron juegos modificados y un torneo de pre-temporada; y, c) 5 minutos de vuelta a la calma, donde se realizaron estiramientos y, a su vez, una reflexión 
grupal de lo trabajado. La cuarta etapa fue la fase final que tenía tres sesiones, donde dos de ellas fueron de competición regular en formato liga, en la que la sesión se estructuró en 10 minutos de calentamiento, 35 minutos de competición, 5 minutos de cumplimentación de informes y, 5 minutos de vuelta a la calma. $A$ continuación, se celebró una última sesión en la que se llevó a cabo el evento final que decidió el puesto de cada equipo, además de realizarse la entrega de diplomas a todo el alumnado.

2.4.2. Fidelidad del modelo. Los dos profesores de EF recibieron un curso formativo de 10 horas de duración sobre los aspectos teóricos y prácticos específicos del modelo pedagógico de ED. Para la estructura y contenido del curso, el equipo investigador se apoyó en los trabajos realizados tanto por Burgueño et al. (2020) como por Gil-Arias et al. (2017). Además de este curso de formación, se realizó una tutela por un equipo investigador experto en la ED. Esta tutela consistió en: análisis de las sesiones, seminarios para la resolución de dudas, inquietudes y problemas y evaluación externa en el centro educativo para verificar que no existían desfases entre el contenido planificado y la practica real. Tales evaluaciones externas fueron respectivamente realizadas por tres investigadores con experiencia en ED quienes confirmaron que las principales características de este modelo pedagógico fueron cumplidas. Para ello, se utilizó la hoja de registro observacional desarrollada por Sinelnikov (2009) y adaptada al contexto español por Calderón et al. (2010).

\subsection{Análisis Estadístico}

La normalidad de los datos fue analizada mediante el coeficiente estandarizado de asimetría y de curtosis. Tales coeficientes estandarizados revelaron valores absolutos menores que 1.96 para el coeficiente de asimetría y de curtosis (ver Tabla 1), lo que sugirió que el supuesto de normalidad no podía ser rechazado (Field, 2017). Los estadísticos descriptivos (media y su desviación típica) fueron estimados para cada una de las variables dependientes examinadas. La consistencia interna de las variables dependientes objeto de estudio fue inspeccionada por medio del coeficiente alfa de Cronbach, aceptable con valores superiores a .70 (Viladrich et al., 2017). Un análisis multivariante de varianza (MANOVA) entre los dos puntos temporales fue aplicado para determinar las posibles diferencias existentes en cada una de las siete actitudes hacia la EF respecto al parámetro temporal. El análisis estuvo controlado por la edad, género, práctica de actividad deportiva extraescolar. El tamaño del efecto fue calculado mediante eta parcial al cuadrado $\left(\eta^{2} p\right)$. Se consideró un efecto del tamaño pequeño con valores menores que 0.01 , medio con valores menores que $0.06 \mathrm{y}$, grande con valores mayores que 0.14 (Field, 2017). El nivel de significación estadístico fue fijado en $p<.05$. El tratamiento estadístico de los datos fue llevado a cabo con el Statistical Package for Social Sciences (IBM SPSS Statistics for Mac, version 25.0; Armonk, NY, USA).

\section{RESULTADOS}

La Tabla 1 muestra ciertos cambios en la puntuación media entre el pre-test y el post-test de cada una de las siete variables dependientes analizadas. De igual 
manera, la Tabla 1 también presenta los valores alfa de Cronbach obtenidos para cada una de las siete variables dependientes consideradas. En concreto, los valores alfa de Cronbach oscilaron entre .71 y .80 en el pre-test $y$, entre .73 y .84 en el post-test, indicando el adecuado nivel de consistencia interna para cada una de las siete variables dependientes objeto de estudio al inicio y al final del programa de intervención.

Tabla I. Estadísticos descriptivos y consistencia interna para cada variable dependiente al inicio y final de la intervención de Educación Deportiva

\begin{tabular}{|c|c|c|c|c|c|c|c|c|c|c|}
\hline & \multicolumn{5}{|c|}{ Pre-test } & \multicolumn{5}{|c|}{ Post-test } \\
\hline & $\alpha$ & $M$ & $D T$ & $\gamma_{1}$ & $\gamma_{2}$ & $\alpha$ & $M$ & $D T$ & $\gamma_{1}$ & $\gamma_{2}$ \\
\hline Valoración de la EF & .73 & 2.83 & 0.44 & 0.16 & 0.17 & .78 & 3.85 & 0.45 & 0.11 & 0.76 \\
\hline Dificultad de la EF & .71 & 2.71 & 0.60 & 0.49 & 0.77 & .73 & 2.91 & 0.61 & 0.37 & 0.28 \\
\hline Utilidad de la EF & .78 & 1.81 & 0.35 & 1.26 & 1.37 & .74 & 3.62 & 0.32 & 0.51 & 0.25 \\
\hline Empatía con Profesor & .80 & 2.35 & 0.70 & 0.02 & 0.54 & .82 & 2.27 & 0.73 & 0.14 & 0.93 \\
\hline Concordancia & .71 & 3.27 & 0.60 & 0.65 & 0.14 & .74 & 3.22 & 0.53 & 0.53 & 0.62 \\
\hline Preferencia por la EF & .72 & 2.08 & 0.63 & 0.49 & 0.02 & .77 & 2.25 & 0.68 & 0.42 & 0.49 \\
\hline EF como Deporte & .79 & 2.36 & 0.75 & 0.46 & 0.52 & .76 & 2.32 & 0.77 & 0.30 & 0.99 \\
\hline
\end{tabular}

Nota: $\mathrm{EF}=$ Educación Física; $\alpha=$ Alfa de Cronbach; $\gamma_{1}=$ Coeficiente Estandarizado de Asimetría; $\gamma_{2}=$ Coeficiente Estandarizado de Curtosis.

En relación con el análisis del parámetro temporal, los resultados derivados de la prueba de MANOVA mostraron un efecto multivariante significativo entre el pre-test y el post-test para el conjunto de las variables dependientes examinadas ( $\lambda$ de Wilks: .68; $F=2.97 ; p=.012 ; \eta^{2}{ }^{2}=0.32$ ). En concreto, se encontró un aumento estadísticamente significativo junto con un tamaño del efecto grande en el nivel tanto de valoración de la EF como de utilidad de la EF. Además, hubo un incremento estadísticamente significativo con un tamaño del efecto medio en el nivel de dificultad de la EF y preferencia por la EF entre el inicio y el final de la intervención de ED. Por otra parte, no se encontraron efectos multivariantes significativos en relación con la edad ( $\lambda$ de Wilks: .96; $F=0.28 ; p=.959 ; \eta^{2}{ }_{p}=$ $0.04)$, práctica deportiva extraescolar ( $\lambda$ de Wilks: $.85 ; F=1.05 ; p=.413 ; \eta^{2}{ }_{\mathrm{p}}=$ 0.15 ) y género ( $\lambda$ de Wilks: .97; $F=0.22 ; p=.980 ; \eta^{2} p=0.03$ ), que ejercieron de variables control del modelo. 
Tabla II. Resultados de la prueba del Análisis Multivariante de Varianza entre el inicio y el final de la intervención de Educación Deportiva para el grupo total y, las variables de control (edad, género y deporte extraescolar)

\begin{tabular}{|c|c|c|c|}
\hline \multicolumn{4}{|l|}{ Grupo total } \\
\hline & $F$ & $p$ & $\eta^{2} p$ \\
\hline Valoración de la Educación Física & 337.58 & $>.001$ & 0.87 \\
\hline Dificultad de la Educación Física & 5.95 & .018 & 0.10 \\
\hline Utilidad de la Educación Física & 1529.01 & $>.001$ & 0.97 \\
\hline Empatía con el Profesor & 1.13 & .292 & 0.02 \\
\hline Concordancia & 0.49 & .487 & 0.01 \\
\hline Preferencia por la Educación Física & 4.55 & .038 & 0.08 \\
\hline Educación Física como Deporte & 0.21 & .653 & 0.01 \\
\hline \multicolumn{4}{|c|}{ Covariable: Edad } \\
\hline & $F$ & $p$ & $\eta^{2} p$ \\
\hline Valoración de la Educación Física & 0.29 & .594 & 0.01 \\
\hline Dificultad de la Educación Física & 1.23 & .272 & 0.03 \\
\hline Utilidad de la Educación Física & 0.12 & .730 & $<0.01$ \\
\hline Empatía con el Profesor & 0.02 & .891 & $<0.01$ \\
\hline Concordancia & 0.16 & .694 & $<0.01$ \\
\hline Preferencia por la Educación Física & 0.44 & .513 & $<0.01$ \\
\hline Educación Física como Deporte & 0.78 & .381 & 0.02 \\
\hline \multicolumn{4}{|c|}{ Covariable: Género } \\
\hline & $F$ & $p$ & $\eta^{2} p$ \\
\hline Valoración de la Educación Física & 0.01 & .946 & $<0.01$ \\
\hline Dificultad de la Educación Física & 0.31 & .580 & 0.01 \\
\hline Utilidad de la Educación Física & 0.12 & .734 & $<0.01$ \\
\hline Empatía con el Profesor & 0.01 & .963 & $<0.01$ \\
\hline Concordancia & 0.17 & .679 & 0.01 \\
\hline Preferencia por la Educación Física & 0.32 & .575 & 0.01 \\
\hline Educación Física como Deporte & 0.24 & .627 & 0.01 \\
\hline \multicolumn{4}{|c|}{ Covariable: Deporte extraescolar } \\
\hline & $F$ & $p$ & $\eta^{2} p$ \\
\hline Valoración de la Educación Física & 1.25 & .269 & 0.03 \\
\hline Dificultad de la Educación Física & 0.15 & .705 & $<0.01$ \\
\hline Utilidad de la Educación Física & 0.01 & .953 & $<0.01$ \\
\hline Empatía con el Profesor & 0.07 & .794 & $<0.01$ \\
\hline Concordancia & 0.01 & .908 & $<0.01$ \\
\hline Preferencia por la Educación Física & 2.06 & .069 & 0.08 \\
\hline Educación Física como Deporte & 0.17 & .685 & $<0.01$ \\
\hline
\end{tabular}

\section{DISCUSIÓN}

El objetivo del estudio fue analizar la influencia de la ED sobre las actitudes hacia la EF en alumnado de educación secundaria obligatoria durante el proceso de enseñanza-aprendizaje deportivo que tiene lugar en EF. Los resultados derivados de esta investigación mostraron que una temporada de ED mejoró significativamente el nivel percibido de la valoración de la EF y de su profesorado, dificultad de la EF, utilidad de la EF y la preferencia por la EF en el alumnado de educación secundaria. 
Acorde con una de las hipótesis propuestas, los resultados derivados de esta investigación revelaron una mejora significativa en el nivel percibido de valoración de la EF y de su profesorado después de la implementación de una temporada de ED. Estos hallazgos se muestran en parte en línea con aquellos obtenidos por Mesquita et al. (2014), en el sentido que el alumnado valoró positivamente el desempeño del profesorado de EF durante la temporada de ED. Posiblemente, el apoyo a la autonomía, que caracteriza a este modelo pedagógico durante su implementación, implicó que el profesorado se convirtiera en un guía para el alumnado y, consecuentemente, el estudiante llegase a ser el centro no sólo de su proceso de enseñanza-aprendizaje sino que también lo fuese para el proceso de enseñanza-aprendizaje de sus compañeros de equipo con quienes comparte su trabajo a lo largo de la temporada (Ginciene \& Matthiesen, 2017; Mesquita et al., 2014). En este sentido, la ED promovió en gran medida que el estudiante asumiera un protagonismo destacado, de acuerdo con su rol desarrollado, en aspectos vinculados con la organización y puesta en práctica de las actividades, el registro de datos, el establecimiento de metas comunes o el feedback. Es más, la compleción de determinados roles como entrenador y árbitro sensibilizó al alumnado de la complejidad de ser docente. Así pues, tanto Mesquita et al. $(2014,2016)$ como Farias et al. (2017) descubrieron la ausencia de una adecuada capacidad de liderazgo por parte de los entrenadores a la hora de optimizar el aprendizaje, cohesión, igualdad de oportunidades y el rendimiento de su equipo, lo que principalmente supuso una dificultad en el desempeño con éxito de su rol al inicio de la temporada. De igual manera, Wahl-Alexander et al. (2017) subrayaron la complejidad de ejercer como árbitro al haber numerosas discrepancias en cuanto a sus decisiones adoptadas durante los partidos. Parece ser que el enfoque centrado en el estudiante propuesto para la ED hizo al alumnado adquirir una visión más real de la labor docente al asumir determinadas decisiones dentro del proceso de enseñanzaaprendizaje, lo que probablemente condujo a una valoración más seria y profunda del papel del profesorado dentro de la clase de EF.

Los resultados emanados del presente estudio indicaron un incremento significativo en el nivel percibido de dificultad de la EF en el alumnado después de una temporada de ED. Una plausible justificación sería que la ED envuelve al alumnado dentro de una experiencia deportiva más auténtica y educativamente más enriquecedora que los tradicionales modelos instructivos utilizados en EF (Araujo et al., 2014), lo que probablemente implicaría un mayor grado de complejidad de la EF percibido por el estudiante. Así pues, el andamiaje curricular de la ED demanda al alumnado entender y poder ejecutar estrategias adecuadas, para hacer frente a la complejidad de la actividad deportiva de manera exitosa por medio del desarrollo de habilidades técnicas y tácticas, la compleción de determinados roles dentro de un mismo equipo, las múltiples interacciones sociales o la resolución de problemas como grupo más que ser simple imitador de la conducta motriz en clase de EF (Hastie \& Wallhead, 2016). Es más, el formato de la ED también exige a los estudiantes no sólo conocer, sino comprender y valorar las reglas, las tradiciones deportivas, así como poder distinguir entre una buena y una mala conducta deportiva con la finalidad de mejorar y proteger la cultura deportiva entre el alumnado (Siedentop, 2002). Todo esto puede hacer que la ED aumente el nivel de dificultad percibido por el estudiante puesto que este modelo pedagógico les ofrece la oportunidad de 
convertirse en plenos deportistas con las herramientas necesarias para analizar críticamente el deporte y, a su vez, comprometerse para cambiarlo (Siedentop, 2002; Siedentop et al., 2020).

En línea con una de las hipótesis planteadas, los resultados obtenidos del actual trabajo mostraron un aumento significativo en el nivel percibido de utilidad de la EF en el alumnado después de una temporada de ED. De igual modo, estos hallazgos se encuentran alineados con una de las principales metas educativas propuestas por Siedentop (2002), en la medida que la ED fue comprendida como un proceso por el cual el deporte podría crecer y prosperar como una influencia humanizadora en las vidas de los ciudadanos. De los hallazgos obtenidos se desprendería que la ED ha sido un modelo pedagógico que ha favorecido, a través del contenido curricular del deporte, la formación integral del alumnado a partir de experiencias deportivas auténticas para chicos y chicas en el ámbito educativo. En un sentido similar, Calderón et al. (2013) destacaron la importancia de la ED en el respectivo desarrollo de las competencias social y ciudadana, aprender a aprender y de autonomía e iniciativa personal en el alumnado como un medio para favorecer la formación integral del estudiante. Una posible explicación sería que el formato de ED tiende esencialmente a promover el desarrollo de habilidades para trabajar en equipo, resolver problemas que se plantean en dichas dinámicas de equipo, así como fomentar el aprendizaje autónomo del alumnado, principalmente en la fase de práctica dirigida por el estudiante, por medio de la constante toma de decisiones en el desarrollo de las actividades para favorecer, tanto la adquisición de conocimientos técnicos y tácticos como la internalización de valores relacionados con la cultura deportiva (Siedentop, 2002).

Los hallazgos encontrados en este estudio reflejaron una mejora significativa en el nivel de preferencia por la EF en el alumnado, lo que apoya una de las hipótesis propuestas para esta investigación. Dichos resultados están en parte alineados con aquellos obtenidos por estudios previos (Calderón et al., 2013; Farias et al., 2017; Mesquita et al., 2014) que mostraron la preferencia del alumnado por la ED a la hora de abordar el proceso de enseñanza-aprendizaje en clase de EF. En concreto, Mesquita et al. (2014) informaron de una elevada alegría en el alumnado cuando entraban en clase de EF porque preferían trabajar en grupos reducidos y tener cierta libertad en la organización de las actividades durante la temporada de ED. Es más, Calderón et al. (2013) matizaron que el alumnado, a pesar de ser instruido con otro modelo pedagógico, continuó manteniendo la inercia pedagógica de la ED como la utilización de los mismos grupos, la misma forma organizativa para la clase y el desempeño de los roles experimentados durante la temporada de ED. En un sentido similar, estos hallazgos son consistentes con la investigación previa (Farias et al., 2018; Méndez-Giménez et al., 2016), en la medida que apuntaron un mayor compromiso e implicación del alumnado en la participación en el juego. Posiblemente, los estudiantes tuvieron una percepción de un aprendizaje más aplicado al contexto deportivo real mediante un trabajo más serio y significativo, mayor interés hacia el formato entrenamiento-competición junto con un mayor conocimiento del deporte y de sus reglas bajo las condiciones de la ED (Calderón et al., 2013; Farias et al., 2017). Es más, quizás el aumento detectado en el nivel de condición física en el alumnado (Wahl-Alexander \& Chomentowski, 2018; 
Ward et al., 2017), acompañado de manera simultánea de una mejora en su nivel de competencia (Cuevas et al., 2015; Mesquita et al., 2016) y de disfrute (Layne \& Hastie, 2016) después de una temporada de ED, pudo suscitar el interés del alumnado hacia la actividad deportiva en el ámbito educativo.

Aunque los resultados de la presente investigación representan un avance en la compresión del andamiaje curricular de la ED en el desarrollo del dominio actitudinal en el alumnado de EF, se deben considerar una serie de limitaciones. La técnica intencional de muestreo, el pequeño número de participantes junto con su amplio rango de edad y la ausencia de grupo control hace interpretar los resultados de manera muy cautelosa, desaconsejando su generalización. Por tanto, se precisan de nuevos trabajos que consoliden o debatan los hallazgos encontrados en esta investigación. Esta investigación fue llevada a cabo con alumnado de educación secundaria obligatoria, por lo que se desconoce el posible efecto de este modelo pedagógico sobre las actitudes hacia la EF en alumnado de distinto nivel educativo. Así pues, futuros trabajos deben examinar la influencia de la ED sobre las actitudes hacia la EF en estudiantes de educación primaria, bachillerato o educación superior. Este trabajo se ha centrado en los deportes alternativos como contenido curricular relacionado con el deporte, por lo que futuras investigaciones deban analizar si deportes más convencionales o juegos pre-deportivos podrían influir sobre las actitudes del alumnado hacia la EF bajo el formato de la ED. Este estudio únicamente ha tomado medidas al inicio y al final de la intervención de ED, lo que ha reducido la inspección de la influencia de este modelo pedagógico sobre las actitudes hacia la EF. De esta manera, nuevos trabajos deben examinar la posible influencia de la ED en cada una de las fases propuestas para este modelo pedagógico con la finalidad de conocer de modo más profundo cómo se produce el cambio actitudinal hacia la EF.

\section{CONCLUSIONES}

Los hallazgos obtenidos en este estudio muestran el aumento significativo de cuatro de las siete actitudes hacia la EF (i.e. valoración de la EF y de su profesorado, dificultad de la EF, utilidad de la EF y preferencia por la EF), descritas por Moreno-Murcia et al. (2003), después de una temporada de ED en la muestra estudiada. Estos resultados podrían implicar que la ED se consideraría un modelo pedagógico apropiado a utilizar por el profesorado de EF para satisfacer las demandas curriculares del alumnado de educación secundaria participante en este estudio en relación con el desarrollo de su dominio actitudinal en clase de EF y, con ello, fomentar la formación integral del estudiante (SHAPE America, 2014). Asimismo, el profesorado de EF, que tomó parte en este trabajo, debería tener en cuenta el empleo de la ED, como un modelo instructivo con la capacidad de promover en el alumnado de educación secundaria las habilidades necesarias, para emitir juicios positivos y estables en relación con los aspectos actitudinales de la EF y, de este modo, contribuir primero al desarrollo del dominio actitudinal del estudiante y, segundo, a su formación integral desde la EF. 


\section{REFERENCIAS BIBLIOGRÁFICAS}

Aicinena, S. (1991). The teacher and student attitudes toward physical education. The Physical Educator, 48, 28-33.

Araujo, R., Mesquita, I., \& Hastie, P. A. (2014). Review of the status of learning in research on Sport Education: Future research and practice. Journal of Sports Science and Medicine, 13(4), 846-858.

Ato, M., López-García, J. J., \& Benavente, A. (2013). A classification system for research designs in psychology. Annals of Psychology, 29(3), 1038-1059. https://doi.org/10.6018/analesps.29.3.178511

Briñol, P., Horcajo, J., Becerra, A., Falces, C., \& Sierra, B. (2002). Cambio de actitudes implícitas. Psicothema, 14(4), 771-775.

Burgueño, R., Cueto-Martín, B., Morales-Ortiz, E., Da Costa Silva, P. C., \& Medina-Casaubón, J. (2018). Clarifying the influence of sport education on basic psychological need satisfaction in high school students. Motricidade, 14(2-3), 48-58. https://doi.org/10.6063/motricidade.13318

Burgueño, R., Cueto-Martín, B., Morales-Ortiz, E., \& Medina-Casaubón, J. (2020). Influence of sport education on high school students' motivational response: A gender perspective. Retos. Nuevas Tendencias En Educación Física $y$ Recreación, 37(1), 604-613. https://doi.org/10.47197/retos.v37i37.70880

Calderón, A., Hastie, P. A., \& Martínez de Ojeda, D. (2010). Learning to teach sport education: Initial experience in elementary education. Cultura, Ciencia y Deporte, 6(5), 169-181. https://doi.org/10.12800/ccd.v5i15.103

Calderón, A., Martínez de Ojeda, D., \& Hastie, P. A. (2013). Students and teachers' perception after practice with two pedagogical models in physical education. RICYDE. Revista Internacional de Ciencias Del Deporte, 32(9), 137-153. https://doi.org/10.5232/ricyde2013.03204

Campbell, D. E. (1969). Student attitudes toward physical education. Research Quarterly. American Association for Health, Physical Education and Recreation, $39(3)$, 456-462. https://doi.org/10.1080/10671188.1968.10616564

Chu, T. L., \& Zhang, T. (2018). Motivational processes of sport education among high school students: A systematic review. European Physical Education Review, 24(3), 372-394. https://doi.org/10.1177/1356336X17751231

Cuevas, R., García-López, L. M., \& Contreras, O. (2015). Influence of the sport education model in the basic psychological needs. Cuadernos de Psicología Del Deporte, 15(2), 155-162.

Digelidis, N., Papaioannou, A., Laparidis, K., \& Chistodoulidis, T. (2003). A oneyear intervention in 7th grade physical education classes aiming to change motivational climate and attitudes towards exercise. Psychology of Sport and Exercise, 4, 195-210. https://doi.org/10.1016/S1469-0292(02)00002-X

Evangelio, C., Sierra-Díaz, J., González-Víllora, S., \& Fernández-Río, J. (2018). The sport education model in elementary and secondary education: A systematic review. Movimento, 24(2), 931-946. https://doi.org/10.22456/1982-8918.81689

Farias, C., Hastie, P. A., \& Mesquita, I. (2017). Towards a more equitable and inclusive learning environment in Sport Education: Results of an action research-based intervention. Sport, Education and Society, 22(4), 460-476. 
https://doi.org/10.1080/13573322.2015.1040752

Farias, C., Mesquita, I., \& Hastie, P. A. (2019). Student game-play performance in invasion games following three consecutive hybrid Sport Education seasons. European Physical Education Review, 25(3), 691-712. https://doi.org/10.1177/1356336X18769220

Farias, C., Valério, C., \& Mesquita, I. (2018). Sport education as a curriculum approach to student learning of invasion games: Effects on game performance and game involvement. Journal of Sports Science and Medicine, 17(1), 56-65. https://doi.org/10.15628/holos.2018.2891

Field, A. (2017). Discoring statistics using IBM SPSS Statistics (5th ed.). SAGE Publications.

Gil-Arias, A., Harvey, S., Cárceles, A., Práxedes, A., \& Del Villar, F. (2017). Impact of a hybrid TGfU-sport education unit on student motivation in physical education. PLOS ONE, 12(6), 1-17. https://doi.org/10.1371/journal.pone.0179876

Gil-Madrona, P., Samalot-Rivera, A., \& Kozub, F. M. (2016). Acquisition and transfer of values and social skills through a physical education program focused in the affective domain. Motricidade, 12(3), 32-38. https://doi.org/10.6063/motricidade.6502

Ginciene, G., \& Matthiesen, S. Q. (2017). The sport education model on the teaching of track and field in school. Movimento, 23(2), 729-742. https://doi.org/10.22456/1982-8918.69788

Gonzalez-Cutre, D., Sicilia, A., Beas-Jiménez, M., \& Hagger, M. S. (2014). Broadening the trans-contextual model of motivation: A study with Spanish adolescents. Scandinavian Journal of Medicine and Science in Sports, 24(4), 306-319. https://doi.org/10.1111/sms.12142

Gülay, O., Mirzeoğlu, D., Çelebi, M., Gulay, O., Mirzeoglu, D., \& Celebi, M. (2010). Effects of cooperative games on social skill levels and attitudes toward physical education. Egitim Arastirmalari - Eurasian Journal of Educational Research, 10(40), 77-92.

Harvey, S., Kirk, D., \& O'Donovan, T. M. (2014). Sport education as a pedagogical application for ethical development in physical education and youth sport. Sport, Education and Society, 19(1), 41-62. https://doi.org/10.1080/13573322.2011.624594

Hastie, P. A., Martínez de Ojeda, D., \& Calderon, A. (2011). A review of research on Sport Education: 2004 to the present. Physical Education and Sport Pedagogy, 16(2), 103-132. https://doi.org/10.1080/17408989.2010.535202

Hastie, P. A., \& Wallhead, T. (2016). Models-based practice in physical education: The case for sport education. Journal of Teaching in Physical Education, 35(4), 390-399. https://doi.org/10.1123/jtpe.2016-0092

Hastie, P. A., Ward, J. K., \& Brock, S. J. (2017). Effect of graded competition on student opportunities for participation and success rates during a season of sport education. Physical Education and Sport Pedagogy, 22(3), 316-327. https://doi.org/10.1080/17408989.2016.1203888

Layne, T. E., \& Hastie, P. A. (2016). Analysis of teaching physical education to second-grade students using sport education. Education 3-13, 44(2), 226240. https://doi.org/10.1080/03004279.2014.914551

Mahedero, P., Calderón, A., Arias-Estero, J. L., Hastie, P. A., \& Guarino, A. J. (2015). Effects of student skill level on knowledge, decision making, skill 
execution and game performance in a mini-volleyball sport education season. Journal of Teaching in Physical Education, 34(4), 626-641. https://doi.org/10.1123/jtpe.2014-0061

Martínez de Ojeda, D., Puente-Maxera, F., \& Méndez-Giménez, A. (2021). Motivational and social effects of a multiannual sport education program. Revista Internacional de Medicina y Ciencias de La Actividad Física y EI Deporte, 21(81),

$29-46$. https://doi.org/https://doi.org/10.15366/rimcafd2021.81.003

Marttinen, R., Fredrick III, R. N., \& Silverman, S. (2018). Changes in student attitude toward physical education across a unit of instruction. Journal of Physical Education and Sport, 18(1), 62-70. https://doi.org/10.7752/jpes.2018.01008

Méndez-Giménez, A., Fernández-Río, J., \& Méndez-Alonso, D. (2015). Sport education model versus traditional model: Effects on motivation and sportsmanship. Revista Internacional de Medicina y Ciencias de La Actividad Física y Del Deporte, 15(59), 449-466. https://doi.org/10.15366/rimcafd2015.59.004

Méndez-Giménez, A., Martínez-de-Ojeda, D., \& Valverde-Pérez, J. J. (2016). Valoración del alumnado y profesorado del material convencional y autoconstruido: estudio longitudinal de diseño cruzado en Educación Deportiva. Retos. Nuevas Tendencias En Educación Física y Recreación, 30, 20-25. https://doi.org/10.47197/retos.v0i30.35725

Mesquita, I., Pereira, C. H., Araújo, R. M., Farias, C. F., Santos, D. F., \& Marques, R. J. (2014). Sport education model: From learning to teaching. Revista Da Educação Física/UEM, 25(1), 1-14. https://doi.org/10.4025/reveducfis.v25i1.21177

Mesquita, I., Rodrigues-Pereira, J. A., Araújo, R., Farias, C., \& Rolim, R. (2016). Representations of the students and their teacher about the educational value of sport education model within an athletic unit. Motricidade, 12(1), 2642. https://doi.org/10.6063/motricidade.4213

Moreno-Murcia, J. A., Rodríguez, P. L., \& Gutiérrez, M. (2003). Intereses y actitudes hacia la educación física. Revista Española de Educación Física, 11(2), 14-28.

Pereira, J., Araújo, R., Farias, C., Bessa, C., \& Mesquita, I. (2016). Sport education and direct instruction units: Comparison of student knowledge development in athletics. Journal of Sports Science and Medicine, 15(4), 569-577.

Pereira, J., Hastie, P. A., Araújo, R., Farias, C., Rolim, R., \& Mesquita, I. (2015). A comparative study of students' track and field technical performance in sport education and in a direct instruction approach. Journal of Sports Science and Medicine, 14(1), 118-127.

SHAPE America- Society of Health and Physical Educators. (2014). National standards \& grade-level outcomes for K-12 physical education. Human Kinetics.

Sicilia, Á., Sáenz-Alvarez, P., González-Cutre, D., \& Ferriz, R. (2015). Analysing the influence of autonomous and controlling social factors within the theory of planned behaviour. Australian Psychologist, 50(1), 70-79. https://doi.org/10.1111/ap.12077

Siedentop, D. (2002). Sport education: A retrospective. Journal of Teaching in 
Physical Education, 21(4), 409-418. https://doi.org/10.1123/jtpe.21.4.409

Siedentop, D., Hastie, P. A., \& van der Mars, H. (2020). Complete guide to sport education (3rd ed.). Human Kinetics.

Sinelnikov, O. A. (2009). Sport education for teachers: Professional development when introducing a novel curriculum model. European Physical Education Review, 15(1), 91-114. https://doi.org/10.1177/1356336X09105213

Viladrich, C., Angulo-Brunet, A., \& Doval, E. (2017). A journey around alpha and omega to estimate internal consistency reliability. Annals of Psychology, 33(3), 755-782. https://doi.org/10.6018/analesps.33.3.268401

Wahl-Alexander, Z., \& Chomentowski, P. (2018). Impact of a university physical conditioning sport education season on students' fitness levels. Health $\begin{array}{llll}\text { Education Journal, } & 77(7), & 828 & -836 .\end{array}$ https://doi.org/10.1177/0017896918776340

Wahl-Alexander, Z., Sinelnikov, O. A., \& Curtner-Smith, M. (2017). A longitudinal analysis of students' autobiographical memories of participation in multiple sport education seasons. European Physical Education Review, 23(1), 2540. https://doi.org/10.1177/1356336X15624246

Ward, J. K., Hastie, P. A., Wadsworth, D. D., Foote, S., Brock, S. J., \& Hollett, N. (2017). A sport education fitness season's impact on students' fitness levels, knowledge, and in-class physical activity. Research Quarterly for Exercise and Sport, 88(3), 346-351. https://doi.org/10.1080/02701367.2017.1321100

Número de citas totales I Total references: 46 (100\%)

Número de citas propias de la revista / Journal's own references: 2 $(4.35 \%)$

Rev.int.med.cienc.act.fís.deporte - vol. 21 - número 83 - ISSN: 1577-0354 